\title{
A POSSIBLE GENERALIZED MODEL OF THE CHLORINE CONCENTRATION DECAY IN PIPES: EXACT SOLUTION
}

\author{
YUSSRI M. MAHROUS* \\ Department of Studies and Basic Sciences, Faculty of Community, University of Tabuk, Saudi Arabia
}

*Corresponding author: y.mahrous@ut.edu.sa; mahrous1972@yahoo.com

\begin{abstract}
This paper discusses a possible generalization of the transport model describing the chlorine concentration decay in pipes. The proposed generalized model is governed by a second-order fractional partial differential equation. The exact solution of the generalized model is obtained via the Laplace transform method and the method of residues. The exact solution reduces to the corresponding published one as the fractional order $\alpha$ tends to one. Analytical expression for the dimensionless cup-mixing average concentration is deduced. Influences of various parameters on the behavior of the dimensionless cup-mixing average concentration are discussed. It is shown that the physical interpretation of the dimensionless cup-mixing average concentration in view of the fractional calculus is completely different than its interpretation in the classical calculus.
\end{abstract}

\section{INTRODUCTION}

Chlorine is the most commonly employed disinfectant in most countries and minimum levels of chlorine must be maintained to ensure the disinfection capacity of distributed water [1,2]. Studying the chlorine decay reflects its importance in engineering and industrial sciences [3]. In this paper, we propose a generalized

Received April 18 ${ }^{\text {th }}, 2021$; accepted May 25 $5^{\text {th }}, 2021$; published June $24^{\text {th }}, 2021$.

2010 Mathematics Subject Classification. 35R11.

Key words and phrases. fractional partial differential equation; Bessel function; boundary value problem; exact solution; Laplace transform.

(C)2021 Authors retain the copyrights of their papers, and all open access articles are distributed under the terms of the Creative Commons Attribution License. 
model of the chlorine transport in pipes. The standard model was formulated by Biswas et. al [4]. The proposed model, in dimensionless form, is governed by the fractional partial differential equation (FPDE):

$$
\frac{\partial^{\alpha} u}{\partial x^{\alpha}}=\frac{A_{0}}{r} \frac{\partial}{\partial r}\left(\frac{1}{r} \frac{\partial u}{\partial r}\right)-A_{1} u, \quad \alpha \in(0,1]
$$

under the boundary conditions (BCs):

$$
\begin{array}{ll}
u(0, r)=1, & 0 \leq r \leq 1, \\
\frac{\partial}{\partial r} u(x, 0)=0, & 0 \leq x \leq 1, \\
\frac{\partial}{\partial r} u(x, 1)+A_{2} u(x, 1)=0, & 0 \leq x \leq 1,
\end{array}
$$

where $u(x, r)$ is the chlorine concentration, $\alpha$ is the order of the fractional derivative in Caputo sense. The dimensionless parameters $A_{0}, A_{1}$ and $A_{2}$ are related to the chlorine decay. The parameter $A_{0}$ stands for the radial diffusion. It depends on the pipe length, the effective diffusivity of chlorine, and the flow rate throughout the system. In addition, $A_{1}$ depends on the reactivity of chlorine with species such as viable cells or chemical compounds in the bulk liquid phase and on the residence time in the system. The parameter $A_{2}$ reflects the wall consumption and depends on the wall consumption rate $V_{d}^{*}$, the pipe radius $r_{0}^{*}$ and the effective diffusivity of chlorine $D$, where $A_{2}=V_{d}^{*} r_{0}^{*} / D$. The objective of this paper is to apply the Laplace transform (LT) method to obtain the exact solution of the system (1)-(4). The LT is a well-known method for solving ordinary differential equations (ODEs) and fractional differential equations (PDEs). Such LT method has been successfully applied on several models such as diffusions [5], heat transfer of nanofluids suspended with carbon-nanotubes [6], singular boundary value problems (SBVPs) related to fluid flow of carbon-nanotubes [7,8], and the MHD Marangoni convection over a flat plate [9]. Furthermore, the LT was successfully applied to solve the Ambartsumian delay equation [10]. Moreover, one can find in Refs. [11-24] other interesting applications of the LT. 


\section{The LT METHOD}

The application of LT, $L(\cdot)$, on both sides of Eq. (1) gives

$$
L\left(\frac{\partial^{\alpha} u}{\partial x^{\alpha}}\right)=L\left(\frac{A_{0}}{r} \frac{\partial}{\partial r}\left(\frac{1}{r} \frac{\partial u(x, r)}{\partial r}\right)\right)-L\left(A_{1} u(x, r)\right)
$$

or

$$
s^{\alpha} U(s, r)-s^{\alpha-1} u(0, r)=\frac{A_{0}}{r} \frac{d}{d r}\left(\frac{1}{r} \frac{d U(s, r)}{d r}\right)-A_{1} U(s, r)
$$

From the BC (2) and Eq. (6), we have

$$
\frac{d^{2} U(s, r)}{d r^{2}}+\frac{1}{r} \frac{d U(s, r)}{d r}-\left(\frac{s^{\alpha}+A_{1}}{A_{0}}\right) U(s, r)=-\frac{s^{\alpha-1}}{A_{0}} .
$$

The solution of Eq. (8) is

$$
U(s, r)=\rho_{1} J_{0}\left(i \sqrt{\frac{s^{\alpha}+A_{1}}{A_{0}}} r\right)+\rho_{2} Y_{0}\left(i \sqrt{\frac{s^{\alpha}+A_{1}}{A_{0}} r}\right)+\frac{s^{\alpha-1}}{s^{\alpha}+A_{1}},
$$

where $i=\sqrt{-1}$. Besides, $J_{0}(\cdot)$ and $Y_{0}(\cdot)$ are Bessel functions and $\rho_{1}$ and $\rho_{2}$ are unknown constants.

Physically, $u(x, r)$ is bounded at $r=0$, hence $\rho_{2}$ must be vanishes and therefore,

$$
U(s, r)=\rho_{1} J_{0}\left(i \sqrt{\frac{s^{\alpha}+A_{1}}{A_{0}} r}\right)+\frac{s^{\alpha-1}}{s^{\alpha}+A_{1}},
$$

and

$$
\frac{d U(s, r)}{d r}=-\rho_{1} i \sqrt{\frac{s^{\alpha}+A_{1}}{A_{0}}} J_{1}\left(i \sqrt{\frac{s^{\alpha}+A_{1}}{A_{0}}} r\right)
$$

where $J_{0}^{\prime}(\lambda r)=-\lambda J_{1}(\lambda r)$. The $\mathrm{BC}(4)$ gives

$$
\frac{d U(s, 1)}{d r}+A_{2} U(s, 1)=0
$$

From Eqs. (9), (10) and (11), we obtain $\rho_{1}$ as

$$
\rho_{1}=-\frac{A_{2} s^{\alpha-1}}{\left(s^{\alpha}+A_{1}\right)\left[A_{2} J_{0}\left(i \sqrt{\frac{s^{\alpha}+A_{1}}{A_{0}}}\right)-i \sqrt{\frac{s^{\alpha}+A_{1}}{A_{0}}} J_{1}\left(i \sqrt{\frac{s^{\alpha}+A_{1}}{A_{0}}}\right)\right]} .
$$


Substituting (12) into (9), yields

$$
U(s, r)=-\frac{A_{2} s^{\alpha-1} J_{0}\left(i \sqrt{\frac{s^{\alpha}+A_{1}}{A_{0}}} r\right)}{\left(s^{\alpha}+A_{1}\right)\left[A_{2} J_{0}\left(i \sqrt{\frac{s^{\alpha}+A_{1}}{A_{0}}}\right)-i \sqrt{\frac{s^{\alpha}+A_{1}}{A_{0}}} J_{1}\left(i \sqrt{\frac{s^{\alpha-1}+A_{1}}{A_{0}}}\right)\right]}+\frac{s^{\alpha-1}}{s^{\alpha}+A_{1}},
$$

which can be written as

$$
U(s, r)=-A_{2} H(s, r)+\frac{s^{\alpha-1}}{s^{\alpha}+A_{1}}
$$

where

$$
H(s, r)=\frac{s^{\alpha-1} J_{0}\left(i \sqrt{\frac{s^{\alpha}+A_{1}}{A_{0}}} r\right)}{\left(s^{\alpha}+A_{1}\right)\left[A_{2} J_{0}\left(i \sqrt{\frac{s^{\alpha}+A_{1}}{A_{0}}}\right)-i \sqrt{\frac{s^{\alpha}+A_{1}}{A_{0}}} J_{1}\left(i \sqrt{\frac{s^{\alpha}+A_{1}}{A_{0}}}\right)\right]} .
$$

Applying the inverse LT on Eq. (14) leads to

$$
u(x, r)=-A_{2} h(x, r)+E_{\alpha}\left(-A_{1} x^{\alpha}\right),
$$

where $h(x, r)$ is the inverse LT of $H(s, r)$ and

$$
h(x, r)=L^{-1}\left(\frac{s^{\alpha-1} J_{0}\left(i \sqrt{\frac{s^{\alpha}+A_{1}}{A_{0}}} r\right)}{\left(s^{\alpha}+A_{1}\right)\left[A_{2} J_{0}\left(i \sqrt{\frac{s^{\alpha}+A_{1}}{A_{0}}}\right)-i \sqrt{\frac{s^{\alpha}+A_{1}}{A_{0}}} J_{1}\left(i \sqrt{\frac{s^{\alpha}+A_{1}}{A_{0}}}\right)\right]}\right) .
$$

\section{The EXACT SOLUtion}

In order to find the exact solution, it should be first evaluate the inverse LT of (17). We observe from

Eq. (15) or Eq. (17) that the denominator has simple poles at $s^{\alpha}=-A_{1}$ and $i \sqrt{\frac{s^{\alpha}+A_{1}}{A_{0}}}=\lambda_{1}, \lambda_{2}, \ldots \lambda_{n}, \ldots$

Hence, we have simple poles at $s=\left(-A_{1}\right)^{1 / \alpha}$ and $s=\left(-A_{1}-A_{0} \lambda_{n}^{2}\right)^{1 / \alpha}, n=1,2,3, \ldots$, where $\lambda_{n}$ are the roots of

$$
A_{2} J_{0}\left(\lambda_{n}\right)-\lambda_{n} J_{1}\left(\lambda_{n}\right)=0 .
$$


So, $h(x, r)$ can be evaluated by applying theorem 1 , in appendix A, by calculating the residues of $e^{s x} H(s, r)$ at $s=\left(-A_{1}\right)^{1 / \alpha}$ and $s=\left(-A_{1}-A_{0} \lambda_{n}^{2}\right)^{1 / \alpha}$, and then by taking their sum. At $s=\left(-A_{1}\right)^{1 / \alpha}$, we have

$$
\begin{aligned}
& \left(\operatorname{Res} e^{s x} H\right)_{s=\left(-A_{1}\right)^{1 / \alpha}}=\lim _{s \rightarrow\left(-A_{1}\right)^{1 / \alpha}} \frac{\left(s-\left(-A_{1}\right)^{1 / \alpha}\right) e^{s x} s^{\alpha-1} J_{0}\left(i \sqrt{\frac{s^{\alpha}+A_{1}}{A_{0}}} r\right)}{\left(s^{\alpha}+A_{1}\right)\left[A_{2} J_{0}\left(i \sqrt{\frac{s^{\alpha}+A_{1}}{A_{0}}}\right)-i \sqrt{\frac{s^{\alpha}+A_{1}}{A_{0}}} J_{1}\left(i \sqrt{\frac{s^{\alpha}+A_{1}}{A_{0}}}\right)\right]} \\
& =e^{\left(-A_{1}\right)^{1 / \alpha} x} \lim _{s \rightarrow\left(-A_{1}\right)^{1 / \alpha}} \frac{s^{\alpha-1} J_{0}\left(i \sqrt{\frac{s^{\alpha}+A_{1}}{A_{0}}} r\right)}{A_{2} J_{0}\left(i \sqrt{\frac{s^{\alpha}+A_{1}}{A_{0}}}\right)-i \sqrt{\frac{s^{\alpha}+A_{1}}{A_{0}}} J_{1}\left(i \sqrt{\frac{s^{\alpha}+A_{1}}{A_{0}}}\right)} \times \\
& \lim _{s \rightarrow\left(-A_{1}\right)^{1 / \alpha}} \frac{s-\left(-A_{1}\right)^{1 / \alpha}}{s^{\alpha}+A_{1}}, \\
& =e^{\left(-A_{1}\right)^{1 / \alpha} x}\left(\frac{\left(-A_{1}\right)^{(\alpha-1) / \alpha} J_{0}(0)}{A_{2} J_{0}(0)-0}\right) \cdot \lim _{s \rightarrow\left(-A_{1}\right)^{1 / \alpha}} \frac{1}{\alpha s^{\alpha-1}}, \\
& =\frac{e^{\left(-A_{1}\right)^{1 / \alpha} x}}{\alpha A_{2}}, \quad \text { where } \quad J_{0}(0)=1 \text {. }
\end{aligned}
$$

At $s=\left(-A_{1}-A_{0} \lambda_{n}^{2}\right)^{1 / \alpha}$, we have

$$
\left(\operatorname{Res} e^{s x} H\right)_{s=\left(-A_{1}-A_{0} \lambda_{n}^{2}\right)^{1 / \alpha}}=\lim _{s \rightarrow\left(-A_{1}-A_{0} \lambda_{n}^{2}\right)^{1 / \alpha}} \frac{\left(s-\left(-A_{1}-A_{0} \lambda_{n}^{2}\right)^{1 / \alpha}\right) e^{s x} s^{\alpha-1} J_{0}\left(i \sqrt{\frac{s^{\alpha}+A_{1}}{A_{0}}} r\right)}{\left(s^{\alpha}+A_{1}\right)\left[A_{2} J_{0}\left(i \sqrt{\frac{s^{\alpha}+A_{1}}{A_{0}}}\right)-i \sqrt{\frac{s^{\alpha}+A_{1}}{A_{0}}} J_{1}\left(i \sqrt{\frac{s^{\alpha}+A_{1}}{A_{0}}}\right)\right]},
$$

or

$$
\begin{aligned}
\left(\operatorname{Res} e^{s x} H\right)_{s=\left(-A_{1}-A_{0} \lambda_{n}^{2}\right)^{1 / \alpha}=} \lim _{s \rightarrow\left(-A_{1}-A_{0} \lambda_{n}^{2}\right)^{1 / \alpha}} \frac{s-\left(-A_{1}-A_{0} \lambda_{n}^{2}\right)^{1 / \alpha}}{A_{2} J_{0}\left(i \sqrt{\frac{s^{\alpha}+A_{1}}{A_{0}}}\right)-i \sqrt{\frac{s^{\alpha}+A_{1}}{A_{0}}} J_{1}\left(i \sqrt{\frac{s^{\alpha}+A_{1}}{A_{0}}}\right)} \times \\
\\
\lim _{s \rightarrow\left(-A_{1}-A_{0} \lambda_{n}^{2}\right)^{1 / \alpha}} \frac{e^{s x} s^{\alpha-1} J_{0}\left(i \sqrt{\frac{s^{\alpha}+A_{1}}{A_{0}}} r\right)}{s^{\alpha}+A_{1}}
\end{aligned}
$$

which can be written as

$$
\left(\operatorname{Res} e^{s x} H\right)_{s=\left(-A_{1}-A_{0} \lambda_{n}^{2}\right)^{1 / \alpha}}=\frac{e^{\left(-A_{1}-A_{0} \lambda_{n}^{2}\right)^{1 / \alpha} x}\left(-A_{1}-A_{0} \lambda_{n}^{2}\right)^{(\alpha-1) / \alpha} J_{0}\left(-\lambda_{n} r\right)}{-A_{0} \lambda_{n}^{2}} . \lim _{s \rightarrow\left(-A_{1}-A_{0} \lambda_{n}^{2}\right)^{1 / \alpha}} Q(s, r) .
$$


Using the L'Hospital's rule, we have

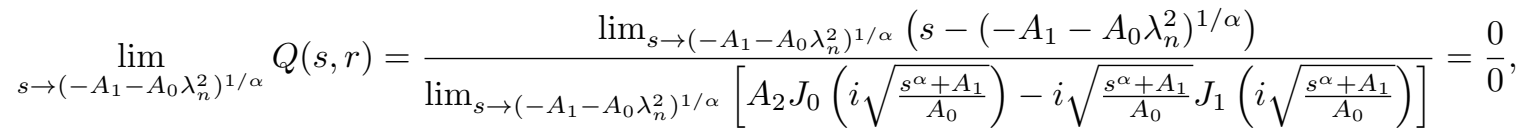

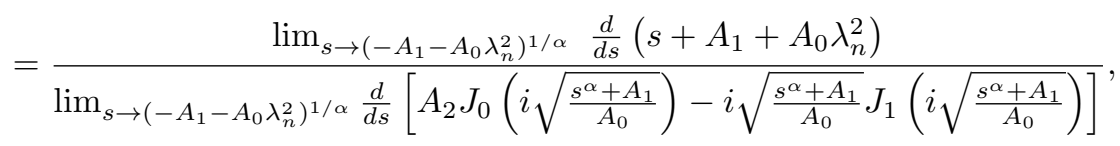

$$
\begin{aligned}
& =\frac{1}{\sigma} \text {, }
\end{aligned}
$$

where $\sigma$ is defined by

$$
\sigma=\lim _{s \rightarrow\left(-A_{1}-A_{0} \lambda_{n}^{2}\right)^{1 / \alpha}} \frac{d}{d s}\left[A_{2} J_{0}\left(i \sqrt{\frac{s^{\alpha}+A_{1}}{A_{0}}}\right)-i \sqrt{\frac{s^{\alpha}+A_{1}}{A_{0}}} J_{1}\left(i \sqrt{\frac{s^{\alpha}+A_{1}}{A_{0}}}\right)\right] .
$$

Assume that $y=i \sqrt{\frac{s^{\alpha}+A_{1}}{A_{0}}}$, then

$$
\begin{aligned}
\sigma & =\lim _{s \rightarrow\left(-A_{1}-A_{0} \lambda_{n}^{2}\right)^{1 / \alpha}} \frac{d}{d s}\left[A_{2} J_{0}(y)-y J_{1}(y)\right] \\
& =\lim _{s \rightarrow\left(-A_{1}-A_{0} \lambda_{n}^{2}\right)^{1 / \alpha}}\left[-A_{2} J_{1}(y) \frac{d y}{d s}-\frac{y}{2}\left(J_{0}(y)-J_{2}(y)\right) \frac{d y}{d s}-J_{1}(y) \frac{d y}{d s}\right], \\
& =\lim _{s \rightarrow\left(-A_{1}-A_{0} \lambda_{n}^{2}\right)^{1 / \alpha}} \frac{d y}{d s}\left[-A_{2} J_{1}(y)-y J_{0}(y)\right],
\end{aligned}
$$

where the properties of Bessel functions are used, see appendix B. The magnitude $\frac{d y}{d s}$ is

$$
\frac{d y}{d s}=-\frac{\alpha s^{\alpha-1}}{2 A_{0} y}
$$

We also note at $s=\left(-A_{1}-A_{0} \lambda_{n}^{2}\right)^{1 / \alpha}$ that

$$
y=-\lambda_{n}, \quad \frac{d y}{d s}=\frac{\alpha\left(-A_{1}-A_{0} \lambda_{n}^{2}\right)^{(\alpha-1) / \alpha}}{2 A_{0} \lambda_{n}} .
$$

Inserting Eqs. (25) into Eq. (23), noting that the functions $J_{0}$ and $J_{2}$ are even and $J_{1}$ is odd, we find

$$
\sigma=\frac{\alpha\left(-A_{1}-A_{0} \lambda_{n}^{2}\right)^{(\alpha-1) / \alpha}}{2 A_{0} \lambda_{n}}\left(A_{2} J_{1}\left(\lambda_{n}\right)+\lambda_{n} J_{0}\left(\lambda_{n}\right)\right)
$$

From (26) and (21), it then follows

$$
\lim _{s \rightarrow\left(-A_{1}-A_{0} \lambda_{n}^{2}\right)^{1 / \alpha}} Q(s, r)=\frac{2 A_{0} \lambda_{n}}{\alpha\left(-A_{1}-A_{0} \lambda_{n}^{2}\right)^{(\alpha-1) / \alpha}\left(A_{2} J_{1}\left(\lambda_{n}\right)+\lambda_{n} J_{0}\left(\lambda_{n}\right)\right)} .
$$


Substituting (27) into (20) and simplifying, gives

$$
\left(\operatorname{Res} e^{s x} H\right)_{s=\left(-A_{1}-A_{0} \lambda_{n}^{2}\right)^{1 / \alpha}}=-\frac{2}{\alpha} \sum_{n=1}^{\infty} \frac{e^{\left(-A_{1}-A_{0} \lambda_{n}^{2}\right)^{1 / \alpha} x} J_{0}\left(\lambda_{n} r\right)}{\lambda_{n}\left[A_{2} J_{1}\left(\lambda_{n}\right)+\lambda_{n} J_{0}\left(\lambda_{n}\right)\right]} .
$$

Hence, $h(x, r)$ in Eq. (17) is given by

$$
\begin{aligned}
h(x, r) & =\left(\operatorname{Res} e^{s x} H\right)_{s=\left(-A_{1}\right)^{1 / \alpha}}+\left(\operatorname{Res} e^{s x} H\right)_{s=\left(-A_{1}-A_{0} \lambda_{n}^{2}\right)^{1 / \alpha},} \\
& =\frac{e^{\left(-A_{1}\right)^{1 / \alpha} x}}{\alpha A_{2}}-\frac{2}{\alpha} \sum_{n=1}^{\infty} \frac{e^{\left(-A_{1}-A_{0} \lambda_{n}^{2}\right)^{1 / \alpha} x} J_{0}\left(\lambda_{n} r\right)}{\lambda_{n}\left[A_{2} J_{1}\left(\lambda_{n}\right)+\lambda_{n} J_{0}\left(\lambda_{n}\right)\right]} .
\end{aligned}
$$

Inserting (29) into (14), and after simplifying, we obtain the solution $u(x, r)$ as

$$
u(x, r)=-\frac{1}{\alpha} e^{\left(-A_{1}\right)^{1 / \alpha} x}+\frac{2}{\alpha} \sum_{n=1}^{\infty} \frac{A_{2} e^{\left(-A_{1}-A_{0} \lambda_{n}^{2}\right)^{1 / \alpha} x} J_{0}\left(\lambda_{n} r\right)}{\lambda_{n}\left[A_{2} J_{1}\left(\lambda_{n}\right)+\lambda_{n} J_{0}\left(\lambda_{n}\right)\right]}+E_{\alpha}\left(-A_{1} x^{\alpha}\right),
$$

Eq. (18) implies

$$
A_{2}=\frac{\lambda_{n} J_{1}\left(\lambda_{n}\right)}{J_{0}\left(\lambda_{n}\right)}
$$

Substituting (31) into (30), yields

$$
u(x, r)=-\frac{1}{\alpha} e^{\left(-A_{1}\right)^{1 / \alpha} x}+\frac{2}{\alpha} \sum_{n=1}^{\infty} \frac{\lambda_{n} J_{1}\left(\lambda_{n}\right) J_{0}\left(\lambda_{n} r\right) e^{\left(-A_{1}-A_{0} \lambda_{n}^{2}\right)^{1 / \alpha} x}}{\left(A_{2}^{2}+\lambda_{n}^{2}\right) J_{0}^{2}\left(\lambda_{n}\right)}+E_{\alpha}\left(-A_{1} x^{\alpha}\right),
$$

As $\alpha \rightarrow 1$, Eq. (32) reduces to

$$
u(x, r)=-e^{-A_{1} x}+2 \sum_{n=1}^{\infty} \frac{\lambda_{n} J_{1}\left(\lambda_{n}\right) J_{0}\left(\lambda_{n} r\right) e^{-\left(A_{1}+A_{0} \lambda_{n}^{2}\right) x}}{\left(A_{2}^{2}+\lambda_{n}^{2}\right) J_{0}^{2}\left(\lambda_{n}\right)}+E_{1}\left(-A_{1} x\right),
$$

which can be simplified to

$$
u(x, r)=2 \sum_{n=1}^{\infty} \frac{\lambda_{n} J_{1}\left(\lambda_{n}\right) J_{0}\left(\lambda_{n} r\right) e^{-\left(A_{1}+A_{0} \lambda_{n}^{2}\right) x}}{\left(A_{2}^{2}+\lambda_{n}^{2}\right) J_{0}^{2}\left(\lambda_{n}\right)}
$$

where $E_{1}\left(-A_{1} x\right)=e^{-A_{1} x}$. The solution (34) is identical to the same result obtained by Biswas et al. [4] for the chlorine decay model with classical partial derivative with respect to $x$. 


\section{Results And Discussion}

According to Biswas et al. [4], the dimensionless cup-mixing average concentration is defined by

$$
u_{\mathrm{av}}=2 \int_{0}^{1} u(x, r) r d r
$$

Substituting (32) into (35), yields

$$
u_{\mathrm{av}}=\left(E_{\alpha}\left(-A_{1} x^{\alpha}\right)-\frac{1}{\alpha} e^{\left(-A_{1}\right)^{1 / \alpha} x}\right) \int_{0}^{1} 2 r d r+4 \sum_{n=1}^{\infty} \frac{\lambda_{n} J_{1}\left(\lambda_{n}\right) e^{\left(-A_{1}-A_{0} \lambda_{n}^{2}\right)^{1 / \alpha} x}}{\left(A_{2}^{2}+\lambda_{n}^{2}\right) J_{0}^{2}\left(\lambda_{n}\right)} \int_{0}^{1} r J_{0}\left(\lambda_{n} r\right) d r
$$

or

$$
u_{\mathrm{av}}=E_{\alpha}\left(-A_{1} x^{\alpha}\right)-\frac{1}{\alpha} e^{\left(-A_{1}\right)^{1 / \alpha} x}+4 \sum_{n=1}^{\infty} \frac{J_{1}^{2}\left(\lambda_{n}\right)}{\left(A_{2}^{2}+\lambda_{n}^{2}\right) J_{0}^{2}\left(\lambda_{n}\right)} e^{\left(-A_{1}-A_{0} \lambda_{n}^{2}\right)^{1 / \alpha} x}
$$

Implementing the relation (31) we have

$$
u_{\mathrm{av}}=E_{\alpha}\left(-A_{1} x^{\alpha}\right)-\frac{1}{\alpha} e^{\left(-A_{1}\right)^{1 / \alpha} x}+4 \sum_{n=1}^{\infty} \frac{A_{2}^{2}}{\lambda_{n}^{2}\left(A_{2}^{2}+\lambda_{n}^{2}\right)} e^{\left(-A_{1}-A_{0} \lambda_{n}^{2}\right)^{1 / \alpha} x}
$$

If the pipe walls act as a perfect sink, i.e., $V_{0}^{*} \rightarrow \infty$ or $A_{2} \rightarrow \infty$, then the cup-mixing average concentration is obtained from Eq. (38) by the limit:

$$
u_{\mathrm{av}}=E_{\alpha}\left(-A_{1} x^{\alpha}\right)-\frac{1}{\alpha} e^{\left(-A_{1}\right)^{1 / \alpha} x}+4 \lim _{A_{2} \rightarrow \infty}\left(\sum_{n=1}^{\infty} \frac{A_{2}^{2}}{\lambda_{n}^{2}\left(A_{2}^{2}+\lambda_{n}^{2}\right)} e^{\left(-A_{1}-A_{0} \lambda_{n}^{2}\right)^{1 / \alpha} x}\right)
$$

which gives

$$
u_{\mathrm{av}}=E_{\alpha}\left(-A_{1} x^{\alpha}\right)-\frac{1}{\alpha} e^{\left(-A_{1}\right)^{1 / \alpha} x}+\sum_{n=1}^{\infty} \frac{4}{\lambda_{n}^{2}} e^{\left(-A_{1}-A_{0} \lambda_{n}^{2}\right)^{1 / \alpha} x},
$$

where $\lambda_{n}$ 's are the roots of $J_{0}\left(\lambda_{n}\right)=0$. Moreover, if $V_{0}^{*} \rightarrow 0$ or $A_{2} \rightarrow 0$ (i.e., the pipe walls are inert and no chlorine consumption takes place at the walls), then $u(x, r)$ in Eq. (19) reduces to

$$
u(x, r)=E_{\alpha}\left(-A_{1} x^{\alpha}\right)
$$

and accordingly,

$$
u_{\mathrm{av}}=2 \int_{0}^{1} E_{\alpha}\left(-A_{1} x^{\alpha}\right) r d r=E_{\alpha}\left(-A_{1} x^{\alpha}\right)
$$


Following Biswas et al. [4], we consider the first three terms of the series (38), hence, three roots $\lambda_{1}, \lambda_{2}$, and $\lambda_{3}$ of Eq. (18) are to be used. Table 1 presents the three roots $\lambda_{1}, \lambda_{2}$ and $\lambda_{3}$ of Eq. (18) at different values of $A_{2}$ in the range $0.01 \leq A_{2}<1$. The roots are calculated using the command "FindRoot" in MATHEMATICA. In Tables 2 and 3, the values of $\lambda_{1}, \lambda_{2}$ and $\lambda_{3}$ are listed for selected values of $A_{2}$ in the range $1 \leq A_{2}<10$ and in the range $10 \leq A_{2}<1000$, respectively.

Table 1. The first three roots $\lambda_{1}, \lambda_{2}$, and $\lambda_{3}$ of Eq. (27) at different values of $A_{2}$ in the range $0.01 \leq A_{2}<1$.

\begin{tabular}{|c|c|c|c|}
\hline$A_{2}$ & $\lambda_{1}$ & $\lambda_{2}$ & $\lambda_{3}$ \\
\hline 0.01 & 0.141245 & 3.83431 & 7.01701 \\
& & & \\
\hline 0.1 & 0.441682 & 3.85771 & 7.02983 \\
\hline 0.2 & 0.616975 & 3.88351 & 7.04403 \\
\hline 0.5 & 0.940771 & 3.95937 & 7.08638 \\
\hline
\end{tabular}

Table 2. The first three roots $\lambda_{1}, \lambda_{2}$, and $\lambda_{3}$ of Eq. (27) at different values of $A_{2}$ in the range $1 \leq A_{2}<10$.

\begin{tabular}{|c|c|c|c|}
\hline$A_{2}$ & $\lambda_{1}$ & $\lambda_{2}$ & $\lambda_{3}$ \\
\hline 1 & 1.25578 & 4.07948 & 7.1558 \\
& & & \\
\hline 2 & 1.59945 & 4.29096 & 7.28839 \\
\hline 5 & 1.98981 & 4.71314 & 7.61771 \\
\hline
\end{tabular}

Table 3. The first three roots $\lambda_{1}, \lambda_{2}$, and $\lambda_{3}$ of Eq. (27) at different values of $A_{2}$ in the range $10 \leq A_{2}<1000$.

\begin{tabular}{|c|c|c|c|}
\hline$A_{2}$ & $\lambda_{1}$ & $\lambda_{2}$ & $\lambda_{3}$ \\
\hline 10 & 2.1795 & 5.03321 & 7.95688 \\
\hline 50 & 2.35724 & 5.4112 & 8.48399 \\
\hline 100 & 2.3809 & 5.46521 & 8.56783 \\
\hline
\end{tabular}

The curves of the cup-mixing average concentration $u_{\text {av }}$ are depicted in Figs. 1-4 versus $A_{1}$, at the outlet $x=1$ of a pipe, for several values of $A_{0}$ and $A_{2}$ when $\alpha=1 / 3$. Figure 1 indicates that the $u_{\mathrm{av}}$ is a decreasing 
function in the parameter $A_{1}$ in the absence of $A_{2}$ (i.e., $A_{2}=0$ ). However, the behavior of $u_{\mathrm{av}}$ is different in the case $A_{2} \neq 0$ where $u_{\text {av }}$ decreases in two subdomians of $A_{1}$ and increases in a certain domain. This last conclusion can be also confirmed and seen in Figs. 2-4 for the curves of $u_{\text {av }}$ when $A_{2}$ has a specified nonezero value, i.e., $A_{2}$ doesn't vanish.

The influence of the fractional order $\alpha$ on the cup-mixing average concentration $u_{\text {av }}$ is displayed in Fig. 5. It can be seen from this figure that $u_{\text {av }}$ is a decreasing function in the full domain of the parameter $A_{1}$ when $\alpha=1$ (classical derivative) of while $u_{\text {av }}$ is of different behavior when $\alpha=\{1 / 3,1 / 5,1 / 7\}$ (fractional derivative). The discussion above may give some lights about the modeling of chlorine decay in view of the fractional calculus.

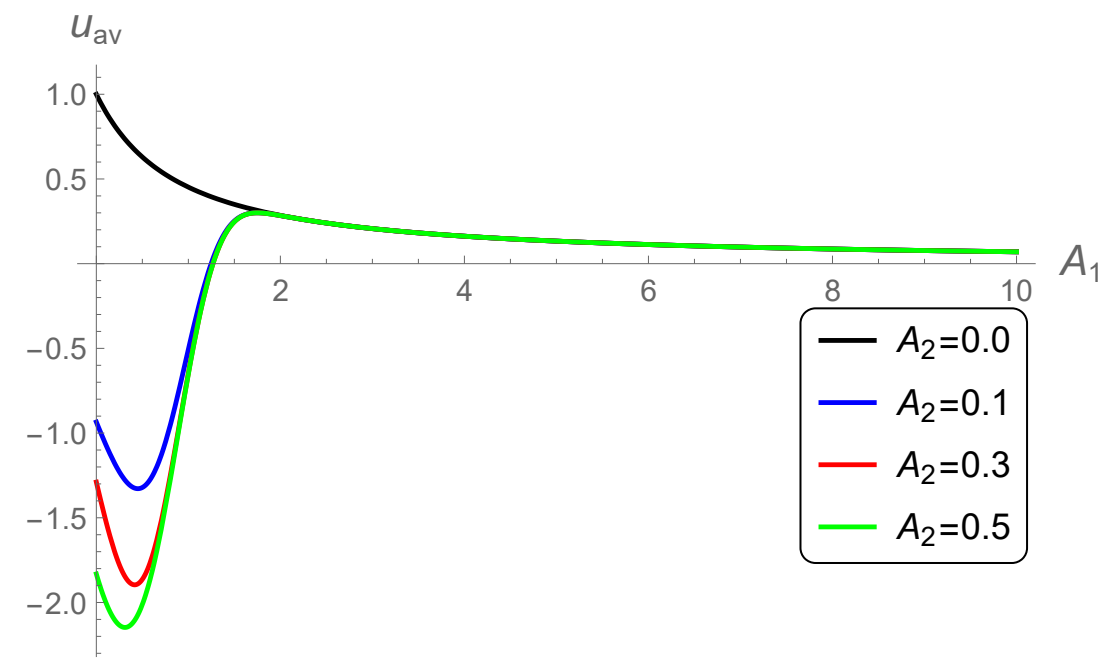

Figure 1. The cup-mixing average concentration $u_{\text {av }}$ versus $A_{1}$ at different values of $A_{2}$ when $\alpha=1 / 3$ and $A_{0}=1.4$. 


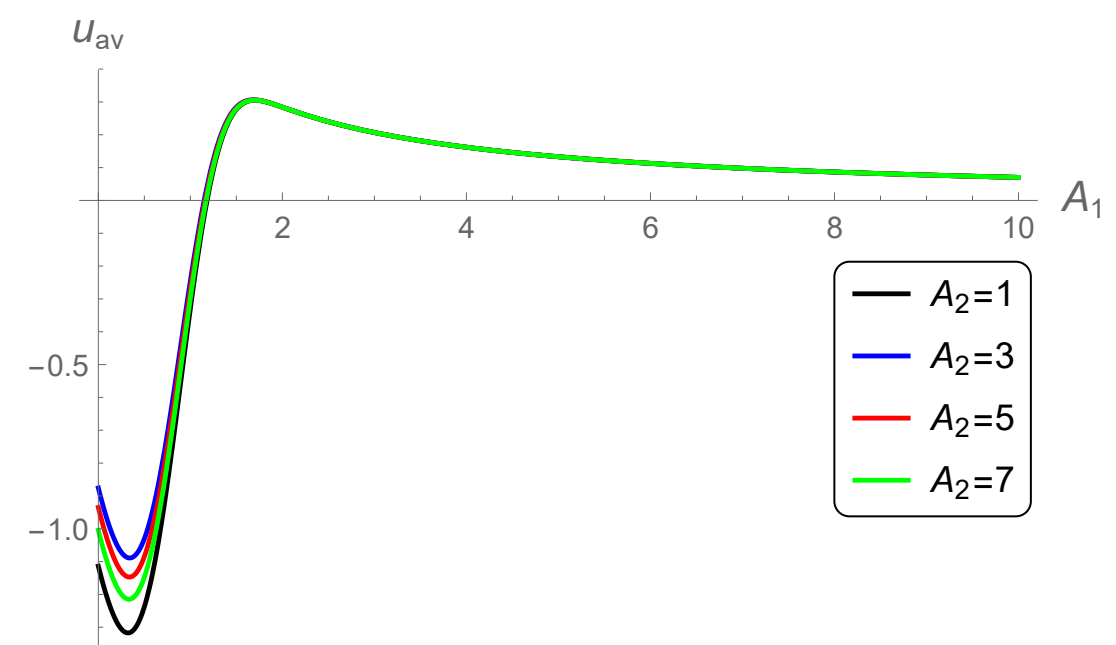

Figure 2. The cup-mixing average concentration $u_{\text {av }}$ versus $A_{1}$ at different values of $A_{2}$ when $\alpha=1 / 3$ and $A_{0}=1.4 \times 10^{-3}$.

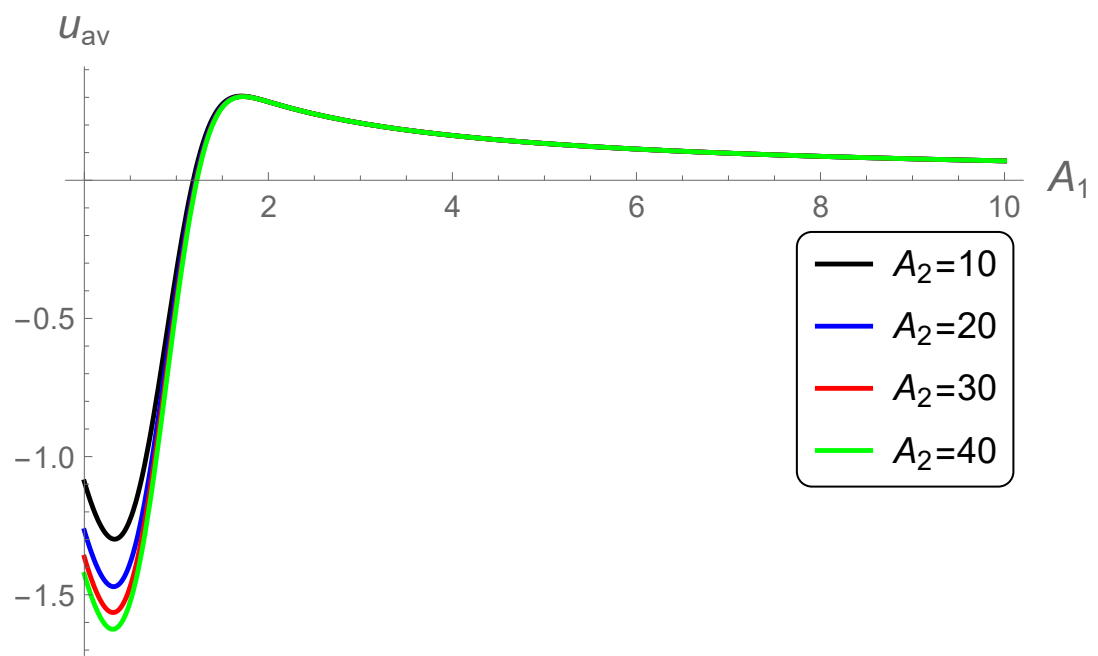

Figure 3. The cup-mixing average concentration $u_{\text {av }}$ versus $A_{1}$ at different values of $A_{2}$ when $\alpha=1 / 3$ and $A_{0}=1.4 \times 10^{-2}$. 


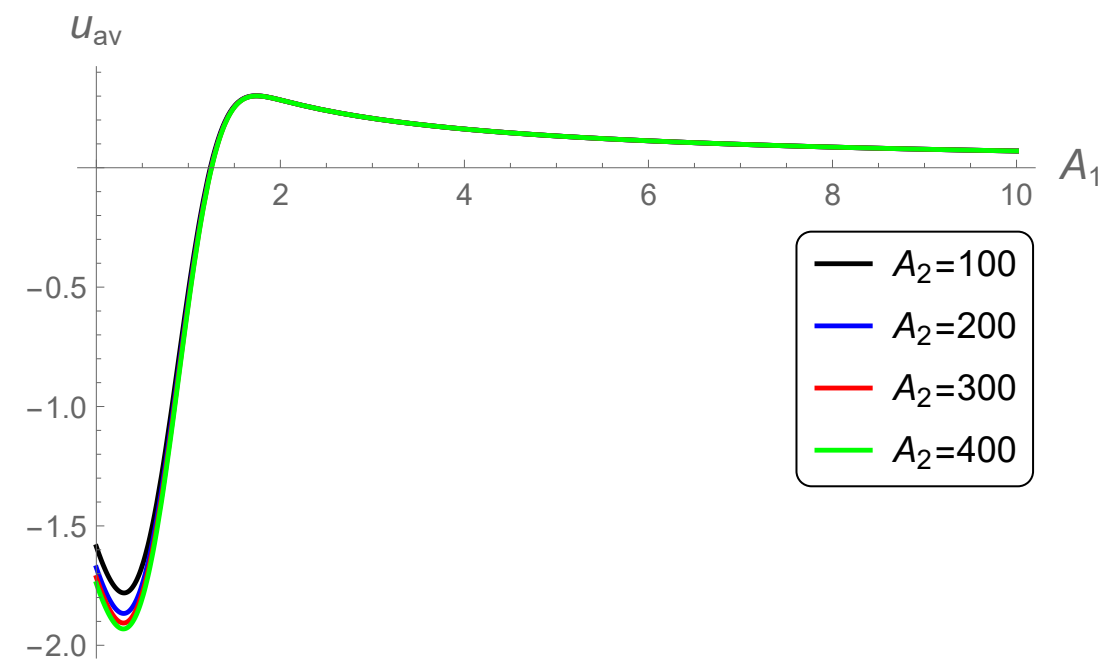

Figure 4. The cup-mixing average concentration $u_{\text {av }}$ versus $A_{1}$ at different values of $A_{2}$ (higher values) when $\alpha=1 / 3$ and $A_{0}=1.4 \times 10^{-2}$.

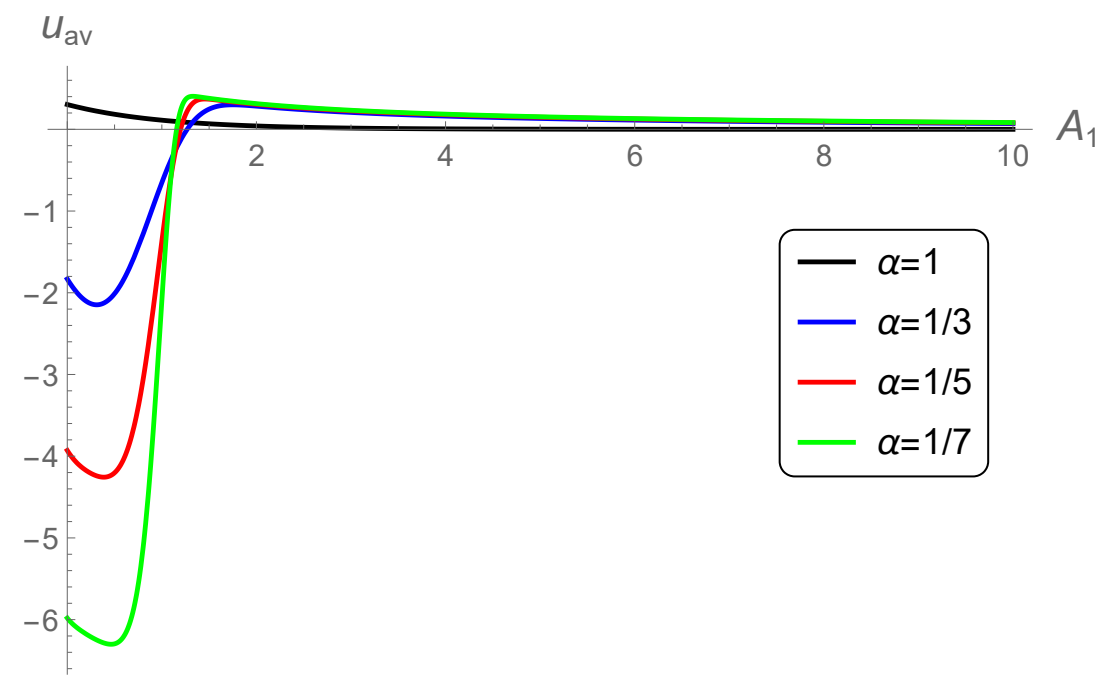

FIGURE 5. Influence of the fractional order $\alpha$ on the cup-mixing average concentration $u_{\text {av }}$ when $A_{0}=1.4$ and $A_{2}=0.5$. 


\section{Conclusion}

A possible generalization of the transport model describing the chlorine concentration decay in pipes was analyzed. The exact solution of the generalized model was obtained using the LT and the method of residues. The obtained exact solutions reduced to the corresponding published solutions as the fractional order $\alpha$ tends to one. Analytical expression for the dimensionless cup-mixing average concentration was deduced. The effects of the impeded parameters on the dimensionless cup-mixing average concentration were discussed and analyzed. The results showed that the behavior of the dimensionless cup-mixing average concentration in view of the fractional calculus is completely different than its behavior using the classical calculus.

\section{Appendices:}

\section{A. Residues method}

A basic theorem for obtaining the inverse LT using the method of residues is given below.

Theorem 1: The inverse LT of a function $H(s, r)$ using the method of residues is given by $h(x, r)=$ Sum of residues of $e^{s x} H(s, r)$ at all poles of $H(s, r)$, see Ref. [25] for details.

\section{B. Properties of Bessel functions}

The Bessel functions $J_{0}(y), J_{1}(y)$, and $J_{2}(y)$ are defined by

$$
\begin{aligned}
& J_{0}(y)=\sum_{k=0}^{\infty} \frac{(-1)^{k}}{(k !)^{2}}\left(\frac{y}{2}\right)^{2 k}, \\
& J_{1}(y)=\sum_{k=0}^{\infty} \frac{(-1)^{k}}{k !(k+1) !}\left(\frac{y}{2}\right)^{2 k+1}, \\
& J_{2}(y)=\sum_{k=0}^{\infty} \frac{(-1)^{k}}{k !(k+2) !}\left(\frac{y}{2}\right)^{2 k+2},
\end{aligned}
$$


and satisfy the properties:

$$
\begin{aligned}
& \frac{d}{d y}\left(J_{0}(\lambda y)\right)=-\lambda J_{1}(\lambda y) \\
& \frac{d}{d y}\left(J_{1}(\lambda y)\right)=\frac{\lambda}{2}\left(J_{0}(\lambda y)-J_{2}(\lambda y)\right), \\
& y J_{2}(y)+y J_{0}(y)=2 J_{1}(y) .
\end{aligned}
$$

Availability of data and materials: Not applicable.

Funding: NA.

Conflicts of Interest: The author(s) declare that there are no conflicts of interest regarding the publication of this paper.

\section{REFERENCES}

[1] R.M. Clark, E.J. Read, J.C. Hoff, Analysis of Inactivation of Giardia Lamblia by Chlorine, J. Environ. Eng. 115 (1989), $80-90$.

[2] M.W. LeChevallier, C.D. Cawthon, R.G. Lee, Inactivation of biofilm bacteria, Appl. Environ. Microbiol. 54 (1988), 2492-2499.

[3] B.F. Arnold, J.M. Colford, Treating Water With Chlorine at Point-of-Use to Improve Water Quality and Reduce Child Diarrhea in Developing Countries: A Systematic Review and Meta-Analysis, Amer. J. Trop. Med. Hyg. 76 (2007), $354-364$.

[4] P. Biswas, C. Lu, R.M. Clark, A model for chlorine concentration decay in pipes, Water Res. 27 (1993), $1715-1724$.

[5] J. Jakubowski, M. Wisniewolski, On matching diffusions, Laplace transforms and partial differential equations, Stoch. Proc. Appl. 125 (2015), 3663-3690.

[6] A. Ebaid, M. Al Sharif, Application of Laplace transform for the exact effect of a magnetic field on heat transfer of carbon-nanotubes suspended nanofluids, Z. Naturforsch., A, 70 (2015), 471-475.

[7] A. Ebaid, A.M. Wazwaz, E. Alali, B. Masaedeh, Hypergeometric Series Solution to a Class of Second-Order Boundary Value Problems via Laplace Transform with Applications to Nanouids, Commun. Theor. Phys. 67 (2017), 231.

[8] A. Ebaid, E. Alali, H. Saleh, The exact solution of a class of boundary value problems with polynomial coefficients and its applications on nanofluids, J. Assoc. Arab Univ. Basi Appl. Sci. 24 (2017), 156-159.

[9] S.M. Khaled, The exact effects of radiation and joule heating on magnetohydrodynamic Marangoni convection over a flat surface, Therm. Sci. 22 (2018), 63-72.

[10] H.O. Bakodah, A. Ebaid, Exact solution of Ambartsumian delay differential equation and comparison with Daftardar-Gejji and Jafari approximate method, Mathematics, 6 (2018), 331.

[11] S. Handibag, B.D. Karande, Laplace Substitution Method for Solving Partial Differential Equations Involving Mixed Partial Derivatives, Int. J. Comput. Eng. Res. 2 (2012), 1049-1052. 
[12] N. Dogan, Solution of the system of ordinary differential equations by combined Laplace transform-Adomian decomposition method, Math. Comput. Appl. 17 (2012), 203-211.

[13] P. Rai, Application of Laplace Transforms to Solve ODE using MATLAB, J. Inform. Math. Sci. 7 (2015), 93-97.

[14] S.S. Handibag, B.D. Karande, Laplace Substitution Method for nth Order Linear and Non-Linear PDE's Involving Mixed Partial Derivatives, Int. Res. J. Eng. Technol. 2 (2015), 378-388.

[15] A.A. Alshikh, M.M.A. Mahgob, A Comparative Study Between Laplace Transform and Two New Integrals "ELzaki" Transform and "Aboodh" Transform, Pure Appl. Math. J. 5 (2016), 145-150.

[16] A. Atangana, B.S.T. Alkaltani, A novel double integral transform and its applications, J. Nonlinear Sci. Appl. 9 (2016), 424-434.

[17] X. Lianga, F. Gao, Y.-N. Gao, X.-J. Yang, Applications of a novel integral transform to partial differential equations, J. Nonlinear Sci. Appl. 10 (2017), 528-534.

[18] P.V. Pavani, U.L. Priya, B.A. Reddy, Solving Differential Equations by using Laplace Transforms, Int. J. Res. Anal. Rev. 5 (2018), 1796-1799.

[19] B.M. Faraj, F.W. Ahmed, On the MATLAB technique by using Laplace transform for solving second order ODE with initial conditions exactly, Matrix Sci. Math. 3 (2019), 8-10.

[20] A. Mousa, T.M. Elzaki, Solution of Volterra Integro-Differential Equations by Triple Laplace Transform, Irish Interdiscip. J. Sci. Res. 3 (2019), 67-72.

[21] R.R. Dhunde, G.L. Waghmare, Double Laplace iterative method for solving nonlinear partial differential equations, New Trends Math. Sci. 7 (2019), 138-149.

[22] D. Ziane, M.H. Cherif, C. Cattani, K. Belghaba, Yang-Laplace Decomposition Method for Nonlinear System of Local Fractional Partial Differential Equations, Appl. Math. Nonlinear Sci. 4 (2019), 489-502.

[23] S. Mastoi, W.A.M. Othman, N. Kumaresan, Randomly generated grids and Laplace Transform for partial differential equations, Int. J. Disaster Recovery Bus. Contin. 11 (2020), 1694-1702.

[24] H. Zhang, M. Nadeem, A. Rauf, Z. Guo Hui, A novel approach for the analytical solution of nonlinear time-fractional differential equations, Int. J. Numer. Meth. Heat Fluid Flow, 31 (2021) 1069-1084.

[25] M.R. Spiegel, Laplace transforms, McGraw-Hill. Inc., New York, 1965. 